\title{
Magnetic Resonance Imaging Criteria to Predict Complete Excision of Parasellar Pituitary Macroadenoma on Postoperative Imaging
}

\author{
S. E. J. Connor ${ }^{1}$ \\ F. Wilson ${ }^{2}$ \\ K. Hogarth ${ }^{3}$ \\ ${ }^{1}$ Department of Neuroradiology, King's College Hospital, London, \\ United Kingdom \\ 2 Department of Paediatric Radiology, Starship Children's Health, \\ Auckland, New Zealand \\ ${ }^{3}$ Department of Neuroradiology, John Radcliffe Hospital, Oxford \\ University Hospitals NHS Trust, Headley Way, Headington, Oxford, \\ United Kingdom
}

J Neurol Surg B 2014;75:41-46.

\begin{abstract}
Address for correspondence S. E. J. Connor, MACP, FRCR, Department of Neuroradiology, Kings College Hospital, Denmark Hill, London SE5 9RS, United Kingdom (e-mail: steve.connor@nhs.net).
\end{abstract}

\begin{abstract}
Purpose To evaluate preoperative magnetic resonance imaging (MRI) criteria for their ability to predict the complete removal of parasellar pituitary macroadenoma on the 3month postoperative MRI.

Methods Dedicated pre-and postoperative pituitary MRI studies were reviewed in 49 patients who had undergone transsphenoidal surgery for macroadenomas with potential unilateral parasellar involvement. Twelve preoperative MRI findings and postoperative MRI outcomes were statistically compared.

Results Depiction of the inferolateral (positive predictive value [PPV]: 0.6; negative predictive value [NPV], 0.92) and lateral (PPV: 0.65; NPV: 0.85) compartments of the cavernous sinus and the percentage of intracavernous carotid artery encasement (PPV: 0.63 ; NPV, 1.0 for $<50 \%$ encasement) were the only criteria significantly predictive of parasellar tumor complete resection. The odds ratios indicated that depiction of the lateral venous or inferolateral venous compartments increased the likelihood of a

Keywords

- magnetic resonance imaging

- pituitary

- macroadenoma

- postoperative complete resection by 6 times, whereas for every 25\% reduction in intracavernous carotid artery encasement, the chance of a complete resection increased 3.4 times. Conclusion The preoperative MR imaging features that are useful in predicting the complete removal of the parasellar component of a pituitary adenoma as assessed by postoperative MRI are (1) depiction of the lateral and inferolateral compartment of the cavernous sinus and (2) decreasing encasement of the intracavernous carotid artery.
\end{abstract}

\section{Introduction}

Transsphenoidal surgical debulking is important in the initial management of most pituitary macroadenomas. However, there is evidence of residual tumor postsurgery in 28 to $70 \%,{ }^{1-4}$ and there is subsequent regrowth in 30 to $50 \%$ of nonfunctioning pituitary adenomas. ${ }^{5-7}$

received

April 6, 2013

accepted after revision

June 18, 2013

published online

August 21, 2013

Pituitary adenomas may extend laterally into the parasellar region, either by displacement of the medial wall of the cavernous sinus or by dural penetration and invasion of the cavernous sinus. Cavernous sinus invasion indicates an aggressive behavior of the tumor, ${ }^{8}$ increases the likelihood of a tumor remnant postsurgery, and reduces the possibility of endocrinological remission in functioning tumors. ${ }^{1,9,10}$

(c) 2014 Georg Thieme Verlag KG Stuttgart · New York
DOI http://dx.doi.org/ 10.1055/s-0033-1353362. ISSN 2193-6331. 
Postoperative magnetic resonance imaging (MRI) is used to detect such residual parasellar tumor. ${ }^{10-12}$ It is conventionally performed at 3 to 4 months after tumor excision, so allowing for the delayed regression of postoperative changes. If a macroscopic tumor remnant is evident on this imaging, then further radiotherapy or pharmacologic therapy is usually advocated to decrease its growth potential. ${ }^{13,14}$

To anticipate the presence of cavernous sinus invasion, various preoperative MRI features have been explored for their ability to predict whether lateral extension of tumor is due to cavernous sinus infiltration rather than displacement. These MRI criteria have been correlated with the presence of cavernous sinus invasion at surgery ${ }^{15-22}$ or whether there is dural infiltration on histology. ${ }^{23}$ Although this is valuable information, it is the presence of residual parasellar tumor on postoperative MRI that dictates the subsequent management and prognosis, rather than evidence of the parasellar invasion itself. Therefore, it is also important for MRI to be able to predict the likelihood of a radical resection, and a so-called radiologic cure of this parasellar tumor would be a more appropriate reference standard. The purpose of this study was to investigate whether preoperative MRI criteria for parasellar extension could be used to indicate whether a complete resection will be subsequently evident on imaging. This will allow earlier consideration and patient discussion concerning the requirement for postoperative radiotherapy or pharmacologic therapy.

\section{Method}

The study was performed at a tertiary neuroscience center with patients managed by a multidisciplinary team and two experienced pituitary surgeons. The study protocol was submitted to the institutional review body and considered as a service evaluation. Consecutive cases with a histologic diagnosis of pituitary adenoma were extracted from a neuropathology database. Imaging and clinical data were used to select those patients who had undergone transsphenoidal surgery for pituitary macroadenoma (between December 2004 and January 2010). The preoperative MRI studies of these patients were initially reviewed. Subjects were selected who demonstrated potential parasellar extension on dedicated preoperative pituitary MRI (3-mm coronal and sagittal pre- and postgadolinium T1-weighted imaging of the pituitary fossa. The preoperative imaging was performed at a mean of 3.75 months (standard deviation [SD]: 3.5 months) prior to surgery. Those cases where parasellar extension was not present were excluded. The absence of parasellar extension was assessed by using MRI criteria, ${ }^{15,21,22}$ which have been shown to have a high negative predictive value for parasellar invasion. These criteria were (1) depiction of the medial venous compartment; (2) percentage encasement of the intracavernous internal carotid artery (ICA) by $<25 \%$, and (3) no crossing of the medial intercarotid line (-Fig. 1). There remained 74 of 354 patients with potential parasellar extension of adenoma on the preoperative MRI. Appropriate postoperative imaging (3-mm coronal and sagittal pre- and postgadolinium T1-weighted imaging of the pituitary fossa

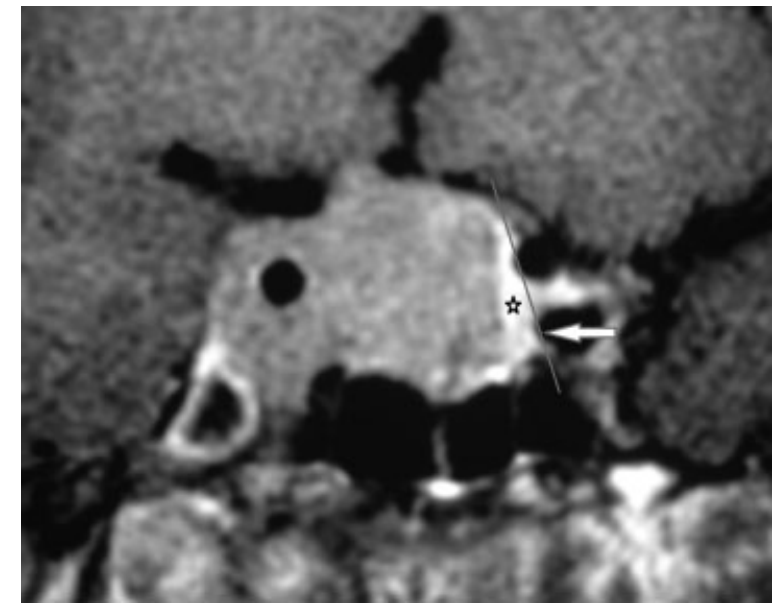

Fig. 1 Gadolinium-enhanced coronal image illustrating criteria used to exclude cases where parasellar extension was not present. On the left there is depiction of the medial venous compartment (star), no encasement of the intracavernous internal carotid artery, and no crossing of the medial intercarotid line (line). On the right there is parasellar extension of adenoma.

at 3 months postsurgery) was only available for 54 of 74 of these patients. It was necessary to exclude a further 5 of 74 patients with bilateral potential parasellar involvement due to reasons of statistical validity, since it was uncertain whether to consider each side as a different entity despite being the same patient. The final group for analysis was composed of 49 patients (28 male, 21 female; mean age: 53 years; age range: 23-85 years; SD age: 15 years).

The MRI studies were reviewed by two neuroradiologists (FW and $\mathrm{KH}$ ), and a consensus was achieved for each recorded outcome. Preoperative MRI studies were scored for the presence or absence of the following criteria based on previous references ${ }^{15,21,22}$ : (1) total encasement of the intracavernous ICA ( - Fig. 1); (2) displacement of the intracavernous ICA by tumor (-Fig. 1); (3) asymmetry of the right and left cavernous sinuses; (4) nondepiction of the lateral sulcus venous compartments (-Fig. 2); (5) nondepiction of the superior venous compartments (-Fig. 2); (6) nondepiction of the inferolateral venous compartments (-Fig. 2); (7) nondepiction of the carotid sulcus venous compartment (-Fig. 2); (8) lateral bulging of the lateral dural wall of the cavernous sinus (convex contour of the wall) (-Fig. 3); (9) whether the tumor crossed one of the intercarotid lines (medial, median, or lateral) according to grade 0 to 3 of the Knosp-Steiner criteria ${ }^{17}$ (-Fig. 2); (10) the perimeter of the intracavernous ICA encased by tumor (divided into twelfths like a clock face) (-Fig. $\mathbf{3}$ ).

The postoperative images were then evaluated for residual disease and classified as either a complete resection or potential residual tumor by consensus, according to a priori criteria. A complete resection was recorded if there was a normally enhancing cavernous sinus (-Fig. 4) or if only a linear plaque of scar tissue was evident, which markedly differed in signal and enhancement to the tumor preoperatively. Potential residual tumor was recorded if (1) there was clear residual parasellar tumor in the same location as that 


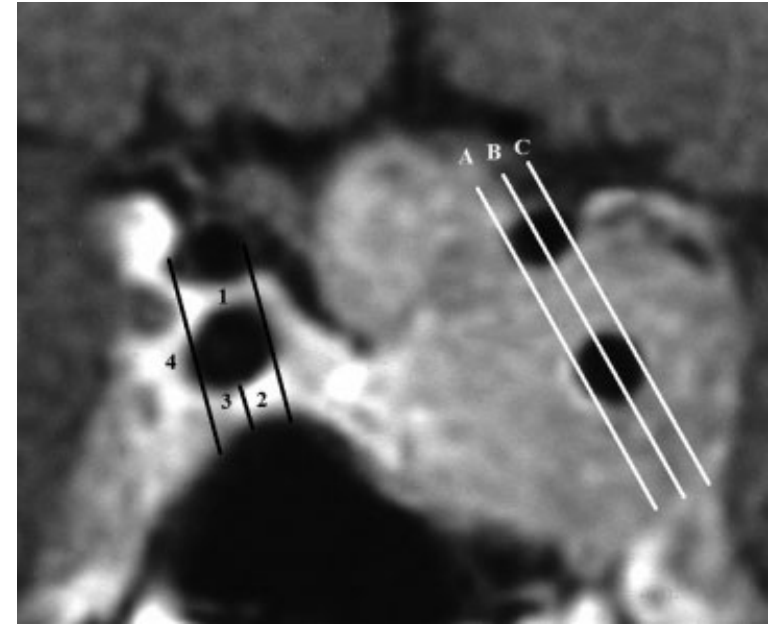

Fig. 2 Gadolinium-enhanced coronal image demonstrating the intercarotid lines and the cavernous sinus venous compartments. The intercarotid lines (left side) were defined as straight lines running between the ipsilateral supraclinoid and cavernous limbs of the carotid artery from their medial edges (medial intercarotid line A), centers (median intercarotid line B), and lateral edges (lateral intercarotid line C). The venous compartments (right side) were superior compartment, 1 ; venous sulcus compartment, 2 ; inferolateral compartment, 3 ; and lateral compartment, 4.

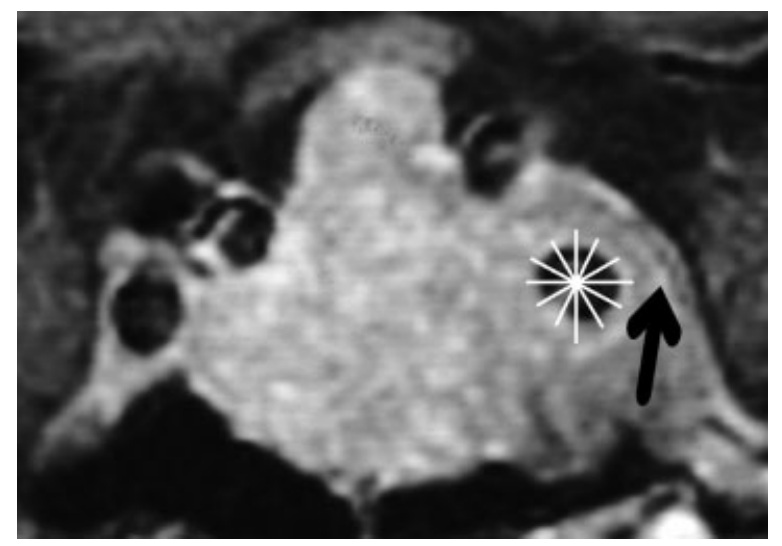

Fig. 3 Gadolinium-enhanced coronal image demonstrating the division of the perimeter of the intracavernous internal carotid artery into twelfths like a clock face, used to score the degree of encasement. There is also bulging of the lateral wall of the cavernous sinus (arrow). shown on the preoperative imaging, and with similar signal and enhancement characteristics (-Fig. 5), or (2) if there was indeterminate abnormal parasellar tissue with signal, enhancement, or location that differed slightly with the tumor on preoperative MRI (-Fig. 6).

Preoperative MRI criteria and the postoperative MRI findings of complete resection versus potential residual tumor were compared using the Fisher exact test. The ORs for potential residual tumor outcomes using each significant criterion were obtained by logistic regression. A two-sample Wilcoxon rank sum test was performed to assess the correlation between the degree of intracavernous ICA encasement and complete resection versus potential residual tumor.

\section{Results}

The postoperative images were evaluated as showing a complete resection in 22 cases and potential residual tumor in 27 cases (of which 3 were indeterminate). - Table 1 shows the correlation between preoperative MRI criteria and the postoperative MRI findings of complete resection versus potential residual tumor. The criteria that reached statistical significance using the Fisher exact test $(p<0.05)$ were the depiction of the inferolateral and lateral compartments of the cavernous sinus. The percentage of the intracavernous carotid encasement was also significantly related to the presence of complete resection versus potential residual tumor as evaluated with a two-sample Mann-Whitney $U$ test $(p<0.0005$; $z=-3.477$ ). Depiction of the lateral or inferolateral venous compartments and the percentage of the intracavernous carotid surrounded by tumor (expressed in quarters) were then used in a logistic regression model. The ORs were 6.1 (95\% confidence interval [CI], 1.28-27.8) for depiction of the lateral venous compartment and 3.4 (95\% CI, 0.98-12.0) for each quarter of intracavernous ICA encasement.

The depiction of the lateral venous compartment, inferolateral venous compartment, and encasement of the intracavernous ICA by $<50 \%$ demonstrated $0.86 / 0.62 / 0.65 / 0.85$, $0.95 / 0.48 / 0.6 / 0.92$ and $1.0 / 0.69 / 0.63 / 1.0$ sensitivity/specificity/positive predictive value/negative predictive value for a complete resection on the postoperative MRI. The high negative predictive values indicate that the adenoma is
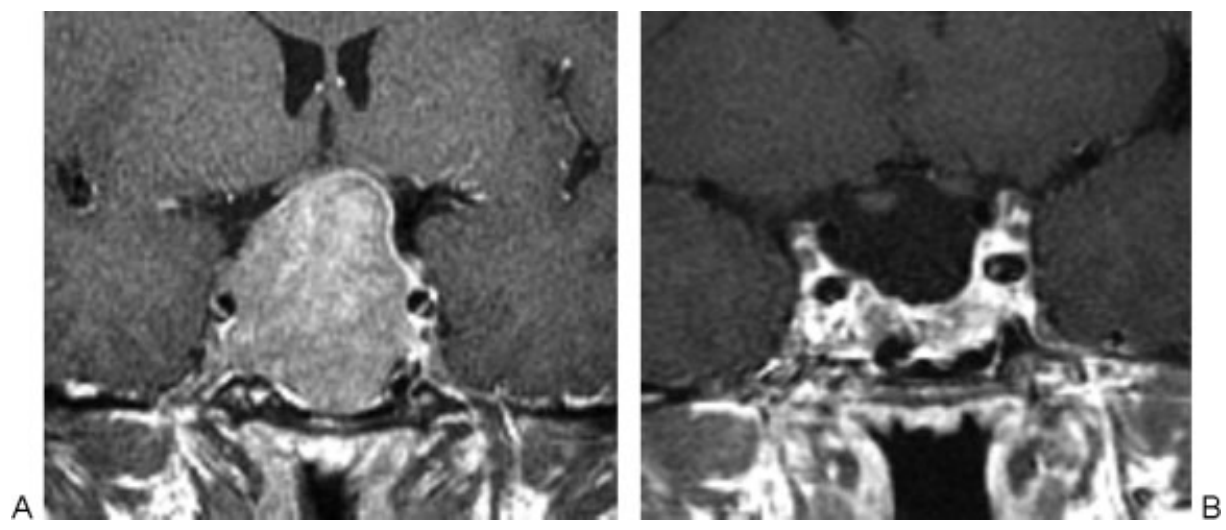

Fig. 4 Gadolinium-enhanced coronal images (A) pre-resection and (B) 3 months postresection. A complete resection was recorded because there is normally enhancing cavernous sinus demonstrated at the site of a preoperative right parasellar tumor. 
A
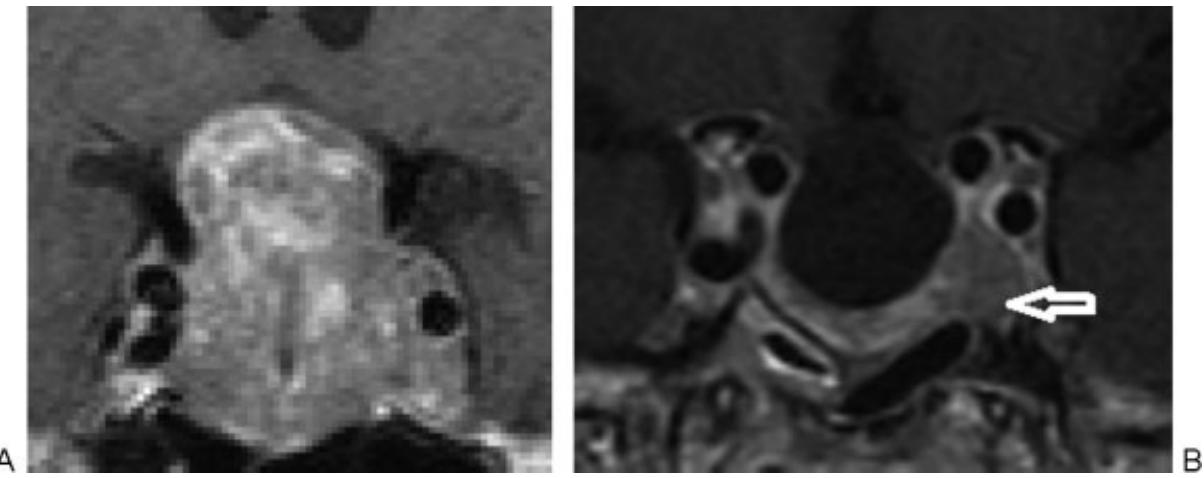

Fig. 5 Gadolinium-enhanced coronal images (A) pre-resection and (B) 3 months postresection. Residual parasellar tumor was recorded because there is clear residual left parasellar tumor in the same location as that shown on the preoperative imaging (arrow), with similar signal and enhancement characteristics.

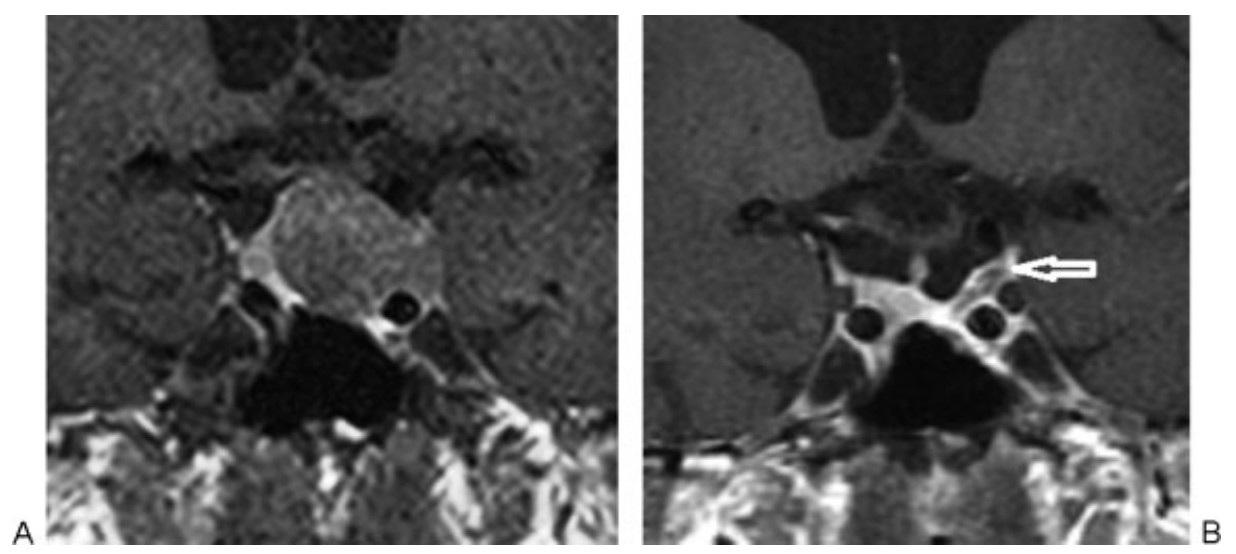

Fig. 6 Gadolinium-enhanced coronal images (A) pre-resection and (B) 3 months postresection. An indeterminate resection was recorded because there is residual tissue that differs slightly to that on preoperative magnetic resonance imaging (arrow) at the site of the preoperative left parasellar tumor.

unlikely to be completely resected if these MRI features are present preoperatively.

\section{Discussion}

Parasellar infiltration by a pituitary adenoma is clinically significant and occurs in 6 to $10 \%$ of cases. ${ }^{24,25}$ The finding indicates increased tumor aggressiveness and renders the tumor a challenging surgical target, with the potential for incomplete resection and hence a requirement for further therapy. Previous studies ${ }^{15-22}$ focused on the ability of MRI criteria to predict cavernous sinus invasion as assessed intraoperatively. Using a surgical reference standard may be imperfect, since the medial wall perforation and exposure

Table 1 Fisher exact scores for the preoperative magnetic resonance imaging criteria versus complete excision/potential residual adenoma

\begin{tabular}{|l|l|}
\hline Magnetic resonance imaging criteria & Fisher exact score \\
\hline Total encasement of cavernous carotid & 0.056 \\
\hline Displacement of cavernous ICA & 0.361 \\
\hline Asymmetry of the cavernous sinuses & 0.51 \\
\hline Nondepiction of the superior compartment of the cavernous sinus & 0.117 \\
\hline Nondepiction of the carotid sulcus compartment of the cavernous sinus & 0.182 \\
\hline Nondepiction of the inferolateral compartment of the cavernous sinus & 0.001 \\
\hline Nondepiction of the lateral compartment of the cavernous sinus & 0.001 \\
\hline Lateral bulging of the lateral dural venous wall of the cavernous sinus & 0.228 \\
\hline Crossing the intercarotid (medial/median/lateral) lines & 0.188 \\
\hline
\end{tabular}

Abbreviation: ICA, internal carotid artery. 
of the intracavernous ICA or intracavernous trabeculae may not be clearly visualized. ${ }^{21}$ Moreover, in terms of the resectability, it may not be the presence of parasellar involvement alone, but rather its extent, which is likely to influence resectability. We therefore aimed to provide objective MRIbased criteria that could stratify cases according to whether there would be radical or subtotal removal of parasellar tumor on postoperative MRI. Although postoperative imaging will still be required, a preoperative assessment of the likelihood of residual parasellar tumor will allow for earlier consideration and preoperative patient discussion concerning the need for additional postoperative therapy.

The assessment of parasellar extension of a macroadenoma is a key part in the evaluation of the preoperative MRI study. There are few indicators of parasellar involvement, since clinical features occur late and subsequent examination of both histologic and molecular tumor markers is inconsistently correlated with parasellar invasion. ${ }^{17,19}$ Since the medial dural wall of the cavernous sinus is very thin and incomplete, it is not possible to directly visualize the penetration of the dura on MRI. ${ }^{26}$ Hence MRI criteria and scoring systems such as the Knosp criteria ${ }^{17}$ have evolved that have been used to predict whether there is infiltration rather than lateral bulging of adenoma into the cavernous sinus. The most specific MRI features shown to predict cavernous sinus invasion at surgery are encroachment on specific venous spaces, encasement of the intracavernous ICA, and crossing of the lateral intercarotid line (according to the Knosp criteria). ${ }^{15-22}$ With respect to the venous spaces, it is nondepiction of the lateral venous compartment ${ }^{22}$ and carotid sulcus venous compartment, ${ }^{15}$ as well as nondepiction of greater than three venous compartments, ${ }^{22}$ which have shown high positive predictive values for cavernous sinus invasion. Our MRI criteria shown to predict residual parasellar tumor on postoperative imaging differ slightly from those used to predict cavernous sinus invasion at the time of surgery. Our study also illustrates the importance of the depiction of the venous compartments; however it was sparing of the most lateral venous spaces (lateral and inferolateral) that was significantly correlated with a complete excision of tumor on postoperative MRI. The likelihood of a radical excision increased by more than six when there was depiction of the normal lateral or inferolateral venous compartments. This finding is biologically plausible because these lateral compartments are the most distant from the pituitary fossa and involvement occurs late, suggesting more advanced disease and a more challenging operation. The degree of intracavernous ICA encasement has also previously been compared with surgical assessment of cavernous sinus invasion using differing criteria. Authors have found percentage encroachments of $25 \%,{ }^{18} 45 \%,{ }^{15} 67 \%,{ }^{22}$ and $100 \%{ }^{16,21}$ to have a high specificity for cavernous sinus invasion. Our data confirm that a reduction in the intracavernous ICA encasement is helpful in anticipating a complete removal of any parasellar tumor. A complete excision is 3.4 times more likely on postoperative imaging with each $25 \%$ reduction in the degree of ICA encasement. Tumor extension beyond the lateral intercarotid line (according to the Knosp criteria) has also proved useful in predicting parasellar inva- sion on prior studies. ${ }^{15,16,18}$ However we did not show this MRI criterion to correlate significantly with postoperative findings of complete excision versus potential residual tumor. Secondary MRI features such as tentorial enhancement have also been proposed as a feature of cavernous sinus compromise. ${ }^{20}$ However these were not explored in this study.

Only one previous study offers some limited data correlating pre- and postoperative MRI appearances following resection of parasellar tumor. ${ }^{1}$ Ramm-Pettersen et al showed that that the mean Knosp grade (progression of parasellar tumor laterally across the intercarotid lines) in tumor that was totally excised on the 3-month postoperative MRI was $3.13(n=20)$; that undergoing subtotal excision was $1.75(n=8)$. Total excision was achieved in all grade 0/1 tumors, $57 \%$ of grade 2 tumors, $8 \%$ of grade 3 tumors, and none of the grade 4 tumors.

Some methodological issues are worthy of comment. The study outcome was intended to be pragmatic. We did not confine the study to patients with confirmed cavernous sinus invasion at surgery, and we included any patient with potential parasellar extension on the basis of previously defined criteria. Similarly, the postoperative MRI evaluation grouped those with clear residual parasellar adenoma alongside those demonstrating indeterminate MRI features. These indeterminate scans were found in three cases. This is a clinical scenario that confronts the radiologist in routine clinical practice, when asked to dichotomize cases as complete excision or not, for the purposes of treatment planning. Some of our indeterminate findings may have been tumor free; however this would be difficult to confirm without systematic reoperation or long-term follow-up without treatment. None of these nonfunctioning tumors showed progression of tumor over 3 years and 4 years on follow-up imaging. Postoperative residual tumor represents a continuum, and it is difficult to define the size of residuum that would prompt intervention, rather than monitoring with serial imaging. Our grouping as complete excision versus potential residual is also justified on the basis of our original hypothesis, which was to determine which MRI features were important in predicting a complete resection, rather than predicting residual tumor, on the postoperative MRI study.

We note that the postoperative MRI assessment required reference to the preoperative imaging that could potentially introduce an element of bias. However this was unavoidable considering our a priori MRI criteria for residual tumor assessment. It is also appreciated that there are likely to be other tumor-related factors that influence the resectability, such as tumor size and consistency. However it was not considered feasible to control for such features. Finally, it is not clear whether our results could be extrapolated to other surgical centers. In particular, differing surgical practices such as the use of endoscopy or intraoperative MRI ${ }^{1,27}$ may improve the rates of resection and alter the predictive value of the various MRI criteria. One study of transsphenoidal surgery aided by intraoperative MRI showed that only 1 of 9 tumors with likely parasellar extension (Knosp grade 3 and 4 tumors) were converted from a subtotal to a total resection when comparing the primary resection with second- and third-look resections, ${ }^{1}$ so the preoperative MRI criteria may remain pertinent in this setting. 
In conclusion, we have determined MR imaging features to be useful in predicting the complete removal of the parasellar component of a pituitary adenoma as assessed by postoperative MRI. These differ slightly from those previously shown to predict cavernous sinus invasion at the time of surgery. The depiction of the lateral and inferolateral compartments and decreasing tumor encasement of the intracavernous ICA are associated with complete resection of parasellar tumor on the postoperative MRI study. In particular, parasellar adenoma is unlikely to be resected completely if there is encasement of $>50 \%$ of the intracavernous ICA or if there is extension to the inferolateral or lateral venous compartments. Patients with these MR imaging features will need to be counseled that residual tumor is likely and additional therapy may be required.

\section{References}

1 Ramm-Pettersen J, Berg-Johnsen J, Hol PK, et al. Intra-operative MRI facilitates tumour resection during trans-sphenoidal surgery for pituitary adenomas. Acta Neurochir (Wien) 2011;153 (7):1367-1373

2 Alameda C, Lucas T, Pineda E, et al. Experience in management of 51 non-functioning pituitary adenomas: indications for postoperative radiotherapy. J Endocrinol Invest 2005;28(1):18-22

3 Ferrante E, Ferraroni M, Castrignanò T, et al. Non-functioning pituitary adenoma database: a useful resource to improve the clinical management of pituitary tumors. Eur J Endocrinol 2006;155(6):823-829

4 Greenman Y, Ouaknine G, Veshchev I, Reider-Groswasser II, Segev Y, Stern N. Postoperative surveillance of clinically nonfunctioning pituitary macroadenomas: markers of tumour quiescence and regrowth. Clin Endocrinol (Oxf) 2003;58(6):763-769

5 Boelaert K, Gittoes NJL. Radiotherapy for non-functioning pituitary adenomas. Eur J Endocrinol 2001;144(6):569-575

6 Ciric I, Mikhael M, Stafford T, Lawson L, Garces R. Transsphenoidal microsurgery of pituitary macroadenomas with long-term followup results. J Neurosurg 1983;59(3):395-401

7 Ebersold MJ, Quast LM, Laws ER Jr, Scheithauer B, Randall RV. Longterm results in transsphenoidal removal of nonfunctioning pituitary adenomas. J Neurosurg 1986;64(5):713-719

8 Martins AN, Hayes GJ, Kempe LG. Invasive pituitary adenomas. J Neurosurg 1965;22:268-276

9 Fahlbusch R, Keller Bv, Ganslandt O, Kreutzer J, Nimsky C. Transsphenoidal surgery in acromegaly investigated by intraoperative high-field magnetic resonance imaging. Eur J Endocrinol 2005;153 (2):239-248

10 Kim M-S, Jang H-D, Kim O-L. Surgical results of growth hormonesecreting pituitary adenoma. J Korean Neurosurg Soc 2009;45 (5):271-274

11 Dina TS, Feaster SH, Laws ER Jr, Davis DO. MR of the pituitary gland postsurgery: serial MR studies following transsphenoidal resection. AJNR Am J Neuroradiol 1993;14(3):763-769
12 Kremer P, Forsting M, Ranaei G, et al. Magnetic resonance imaging after transsphenoidal surgery of clinically non-functional pituitary macroadenomas and its impact on detecting residual adenoma. Acta Neurochir (Wien) 2002;144(5):433-443

13 Bradley KM, Adams CB, Potter CP, Wheeler DW, Anslow PJ, Burke $\mathrm{CW}$. An audit of selected patients with non-functioning pituitary adenoma treated by transsphenoidal surgery without irradiation. Clin Endocrinol (Oxf) 1994;41(5):655-659

14 Turner HE, Stratton IM, Byrne JV, Adams CBT, Wass JAH. Audit of selected patients with nonfunctioning pituitary adenomas treated without irradiation-a follow-up study. Clin Endocrinol (Oxf) 1999;51(3):281-284

15 Cottier J-P, Destrieux C, Brunereau L, et al. Cavernous sinus invasion by pituitary adenoma: MR imaging. Radiology 2000;215(2):463-469

16 Knosp E, Kitz K, Steiner E, Matula C. Pituitary adenomas with parasellar invasion. Acta Neurochir Suppl (Wien) 1991;53:65-71

17 Knosp E, Steiner E, Kitz K, Matula C. Pituitary adenomas with invasion of the cavernous sinus space: a magnetic resonance imaging classification compared with surgical findings. Neurosurgery 1993;33(4):610-617; discussion 617-618

18 Moreau L, Cottier JP, Bertrand P, et al. MRI diagnosis of sinus cavernous invasion by pituitary adenomas [in French]. J Radiol 1998;79(3):241-246

19 Pan LX, Chen ZP, Liu YS, Zhao JH. Magnetic resonance imaging and biological markers in pituitary adenomas with invasion of the cavernous sinus space. J Neurooncol 2005;74(1):71-76

20 Nakasu Y, Nakasu S, Ito R, Mitsuya K-I, Fujimoto O, Saito A. Tentorial enhancement on MR images is a sign of cavernous sinus involvement in patients with sellar tumors. AJNR Am J Neuroradiol 2001;22(8):1528-1533

21 Scotti G, Yu CY, Dillon WP, et al. MR imaging of cavernous sinus involvement by pituitary adenomas. AJR Am J Roentgenol 1988;151(4):799-806

22 Vieira JO Jr, Cukiert A, Liberman B. Evaluation of magnetic resonance imaging criteria for cavernous sinus invasion in patients with pituitary adenomas: logistic regression analysis and correlation with surgical findings. Surg Neurol 2006;65(2):130-135; discussion 135

23 Daita G, Yonemasu Y, Nakai H, Takei H, Ogawa K. Cavernous sinus invasion by pituitary adenomas-relationship between magnetic resonance imaging findings and histologically verified dural invasion. Neurol Med Chir (Tokyo) 1995;35(1):17-21

24 Ahmadi J, North CM, Segall HD, Zee CS, Weiss MH. Cavernous sinus invasion by pituitary adenomas. AJNR Am J Neuroradiol 1985;6: 893-898

25 Fahlbusch R, Buchfelder M. Transsphenoidal surgery of parasellar pituitary adenomas. Acta Neurochir (Wien) 1988;92(1-4):93-99

26 Lonser RR, Ksendzovsky A, Wind JJ, Vortmeyer AO, Oldfield EH. Prospective evaluation of the characteristics and incidence of adenoma-associated dural invasion in Cushing disease. J Neurosurg 2012;116(2):272-279

27 Bohinski RJ, Warnick RE, Gaskill-Shipley MF, et al. Intraoperative magnetic resonance imaging to determine the extent of resection of pituitary macroadenomas during transsphenoidal microsurgery. Neurosurgery 2001;49(5):1133-1143; discussion $1143-1144$ 